\title{
Pre-flight optical test and calibration for the Cosmic Infrared Background ExpeRiment 2 (CIBER-2)
}

Takimoto, Kohji, Bang, Seung-Cheol, Bangale, Priyadarshini, Bock, James, Cooray, Asantha, et al.

Kohji Takimoto, Seung-Cheol Bang, Priyadarshini Bangale, James J. Bock, Asantha Cooray, Kenta Danbayashi, Richard M. Feder, Masaki Furutani, Kevin Gates, Ryo Hashimoto, Viktor Hristov, Arisa Kida, Phillip Korngut, Alicia Lanz, Dae-Hee Lee, Lunjun Liu, Peter Mason, Toshio Matsumoto, Shuji Matsuura, Jodi-Ann Morgan, Chi H. Nguyen, Won-Kee Park, Dorin Patru, James Parkus, Shohta Sakai, Kei Sano, Hiroko Suzuki, Aoi Takahashi, Sohta Tatsu, Kohji Tsumura, Takehiko Wada, Shiang-Yu Wang, Yasuhiro Yamada, Michael Zemcov, "Pre-flight optical test and calibration for the Cosmic Infrared Background ExpeRiment 2 (CIBER-2)," Proc. SPIE 11443, Space Telescopes and Instrumentation 2020: Optical, Infrared, and Millimeter Wave, 114435A (13 December 2020); doi: 10.1117/12.2561917 


\title{
Pre-flight Optical Tests and Calibration for the Cosmic Infrared Background ExpeRiment 2 (CIBER-2)
}

Kohji Takimoto $^{\mathrm{a}}$, Seung-Cheol Bang ${ }^{\mathrm{b}}$, Priyadarshini Bangale ${ }^{\mathrm{c}}$, James J. Bock ${ }^{\mathrm{d}, \mathrm{e}}$, Asantha Cooray $^{f}$, Kenta Dambayashi ${ }^{\mathrm{a}}$, Richard M. Feder ${ }^{\mathrm{d}}$, Masaki Furutani ${ }^{\mathrm{a}}$, Kevin Gates ${ }^{\mathrm{c}}$, Ryo Hashimoto $^{\mathrm{a}}$, Viktor Hristov ${ }^{\mathrm{d}}$, Arisa Kida ${ }^{\mathrm{a}}$, Phillip Korngut ${ }^{\mathrm{d}}$, Alicia Lanz ${ }^{\mathrm{g}}$, Dae-Hee Lee ${ }^{\mathrm{b}}$, Lunjun Liu ${ }^{\mathrm{d}}$, Peter Mason ${ }^{\mathrm{d}}$, Toshio Matsumoto,i, Shuji Matsuura ${ }^{\mathrm{a}}$, Jodi-Ann Morgan ${ }^{\mathrm{c}}$, Chi H. Nguyen $^{c}$, Won-Kee Park ${ }^{b}$, Dorin Patru ${ }^{c}$, James Parkus ${ }^{c}$, Shohta Sakai ${ }^{\mathrm{a}}$, Kei Sano ${ }^{\mathrm{j}}$, Hiroko Suzuki $^{\mathrm{a}}$, Aoi Takahashik ${ }^{\mathrm{k}}$, Sohta Tatsu ${ }^{\mathrm{a}}$, Kohji Tsumural, Takehiko Wada ${ }^{\mathrm{h}}$, Shiang-Yu Wangi, Yasuhiro Yamada ${ }^{a}$, and Michael Zemcove,e

aSchool of Science and Technology, Kwansei Gakuin University, Sanda, Hyogo 669-1337, Japan; ${ }^{\mathrm{b}}$ Korea Astronomy and Space Science Institute (KASI), Daejeon 305-348, Republic of Korea; ${ }^{\mathrm{c}}$ Center for Detectors, Rochester Institute of Technology, Rochester, New York 14623, USA;

${ }^{\mathrm{d}}$ Department of Physics, Mathematics and Astronomy, California Institute of Technology, Pasadena, California 91125, USA;

e Jet Propulsion Laboratory (JPL), National Aeronautics and Space Administration (NASA), Pasadena, California 91109, USA;

${ }^{\mathrm{f}}$ Department of Physics \& Astronomy, University of California, Irvine, California 92697, USA; ${ }^{\text {g}}$ The Observatories of the Carnegie Institution for Science, Pasadena, California 91101, USA; ${ }^{\mathrm{h}}$ Institute of Space and Astronautical Science (ISAS), Japan Aerospace Exploration Agency (JAXA), Sagamihara, Kanagawa 252-5210, Japan;

${ }^{i}$ Institute of Astronomy and Astrophysics, Academia Sinica, Taipei 10617, Taiwan R. O. C.; ${ }^{j}$ College of Science and Engineering, School of Mathematics and Physics, Kanazawa University, Kakuma, Kanazawa, Ishikawa 920-1192, Japan;

${ }^{\mathrm{k}}$ Astrobiology Center, National Institutes of Natural Sciences, Mitaka, Tokyo 181-8588, Japan; ${ }^{1}$ Department of Natural Science, Faculty of Science and Engineering, Tokyo City University, Setagaya, Tokyo 158-8557, Japan

\begin{abstract}
The total integrated emission from galaxies, known as the Extragalactic Background Light (EBL), is an important observable for understanding the history of star formation over the history of the universe. Spatial fluctuations in the infrared EBL as measured by the Cosmic Infrared Background ExpeRiment (CIBER), Spitzer and AKARI exceed the predicted signal from galaxy clustering alone. The CIBER-2 project seeks to extend CIBER observations of the EBL throughout the near infrared into the optical, through measurements above Earth's atmosphere during a suborbital sounding rocket flight. The experiment has a LN2-cooled $28.5 \mathrm{~cm}$ Cassegrain telescope along with three optical paths and dichroic beamsplitters, which are used to obtain three wide-field images in six broad spectral bands between 0.5-2.0 $\mu \mathrm{m}$. The three focal planes also contain linear variable filters (LVFs) which simultaneously take spectra with resolution $\mathrm{R}=20$ across the same range. CIBER-2 is scheduled to fly multiple times on a Black Brant IX sounding rocket from White Sands Missile Range in the New Mexico desert. For the first flight, scheduled for early 2021, we have completed a variety of pre-flight optical tests, which we use to make focus adjustments, spectral response measurements, and absolute photometric calibrations. In this paper, we describe the methods behind these tests and present their results for pre-flight performance evaluation. In particular, we present measurements of the PSF for each broad spectral band, along with absolute calibration
\end{abstract}

Further author information: (Send correspondence to K.T.)

K.T.: E-mail: takimoto.koji@kwansei.ac.jp

Space Telescopes and Instrumentation 2020: Optical, Infrared, and Millimeter Wave, edited by Makenzie Lystrup, Marshall D. Perrin, Proc. of SPIE Vol. 11443, 114435A · @ 2020 SPIE

CCC code: $0277-786 \mathrm{X} / 20 / \$ 21 \cdot$ doi: $10.1117 / 12.2561917$ 
factors for each band and the LVF. Through monochromator scans, we also measure the spectral responsivity of each LVF as a function of position.

Keywords: Cosmology, Extragalactic astronomy, CIBER-2, Cosmic infrared background, Sounding rocket experiment

\section{INTRODUCTION}

The Extragalactic Background Light (EBL) is defined as the integrated radiation produced by stellar nucleosynthesis and gravitational accretion over cosmic history. The EBL contains contributions from the first generation of stars and galaxies formed during the Epoch of Reionization (EoR) and can be used to constrain cosmological models of structure formation. ${ }^{1}$ Spatial fluctuations of the near-infrared EBL as measured by the Cosmic Infrared Background ExpeRiment (CIBER), ${ }^{2}$ Spitzer $^{3,4}$ and AKARI ${ }^{5,6}$ exceed that predicted from galaxy clustering. ${ }^{7,8}$ Possible sources for excess fluctuations are new foreground components, such as color variations in the Zodiacal light, or new EBL components due to unaccounted emission from galaxies, such as EoR and Intra Halo Light (IHL). IHL is extended, low surface brightness emission produced by tidal streams of stars due to gravitational interactions during galaxy formation at $0<z<2 .^{3}$

Further observational studies in a wider wavelength range are needed to improve the accuracy of the EBL measurements, due to the uncertainties with the ZL subtraction. The wavelength coverage also helps with probing the Lyman break of EoR galaxies and the colors of IHL. As evident in Figure 1, measurements from the infrared to the optical are important both to fully test the IHL model and to search for the EoR component. In the optical, EoR emission is negligible due to Lyman absorption at $z \sim 7$, while IHL emission from low-mass stars peaks around $1 \mu \mathrm{m}$ and slowly decreases.

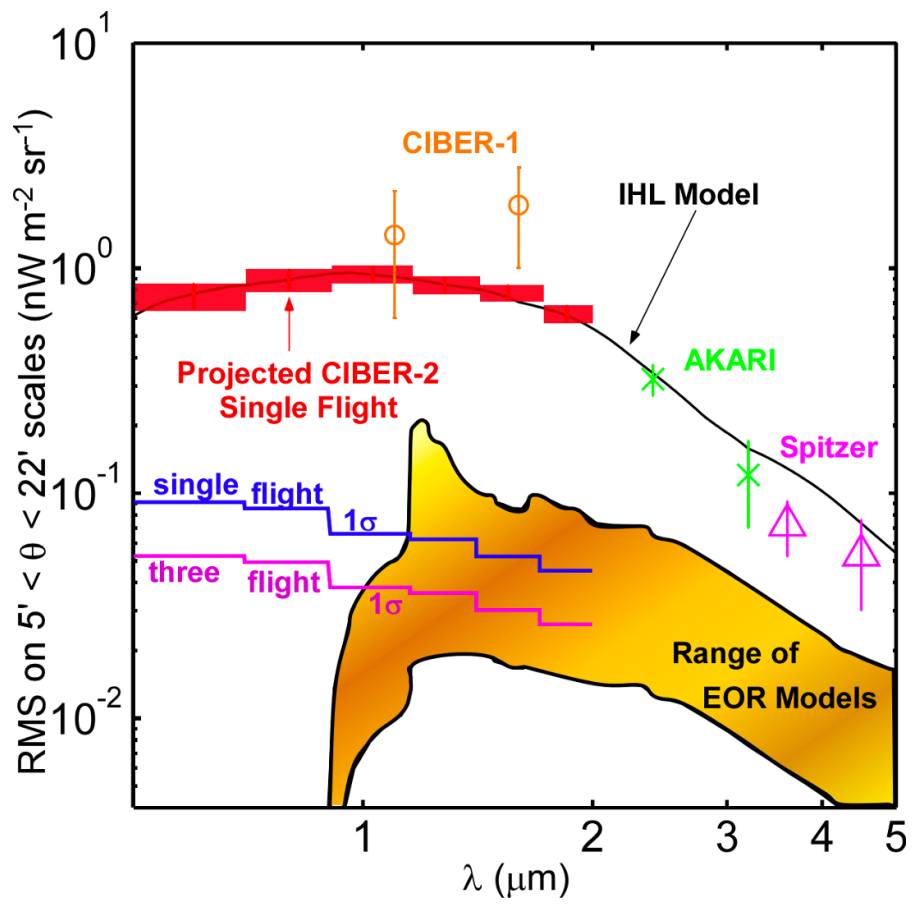

Figure 1. The electromagnetic spectrum of EBL. The measured amplitude of CIBER, Spitzer, and Akari fluctuations follow a blue, nearly Rayleigh-Jeans spectrum from 1-4 $\mu \mathrm{m}$, fitted with an IHL model spectrum. The amplitude of EoR fluctuations is modelled to lie in the orange shaded region. CIBER-2 will make high-accuracy measurements of EBL anisotropy that will inform the history of IHL production (projected CIBER-2 errors given in red), and will further constrain the fainter EoR component. 
The Cosmic Infrared Background ExpeRiment 2 (CIBER-2) is designed to follow up on CIBER's initial detection of excess fluctuations by extending observations from the near infrared into the optical, through measurements above Earth's atmosphere during a suborbital sounding rocket flight. CIBER-2 is scheduled to fly multiple times on a Black Brant IX sounding rocket from White Sands Missile Range in the New Mexico desert. We have completed a variety of pre-flight optical tests, and CIBER-2 is currently qualified for the first flight scheduled for early 2021. in this paper, we describe the instrument detail in Section 2, and the methods behind optical tests and results for pre-flight performance evaluation in Section 3.

\section{INSTRUMENT OVERVIEW}
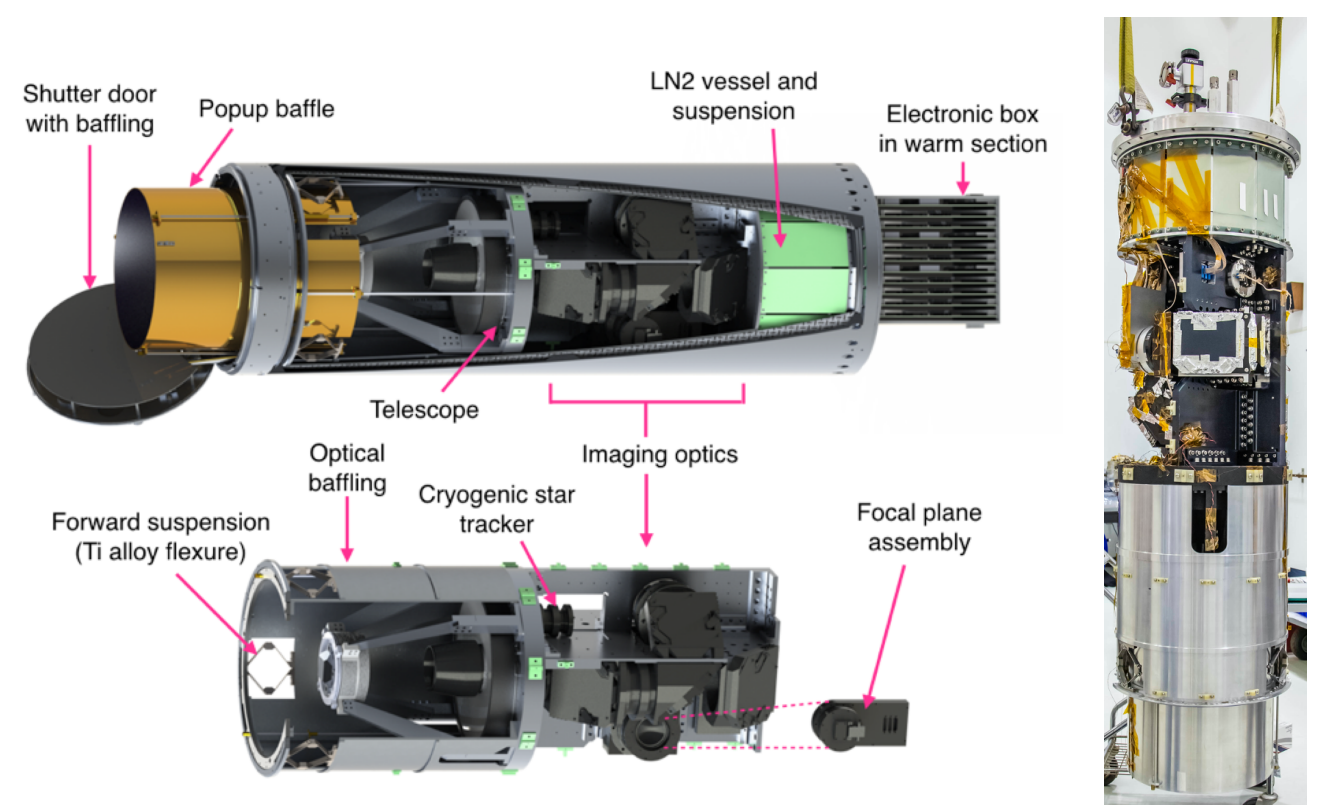

Figure 2. Schematics (left) and photograph (right) of the CIBER-2 payload. The rocket skin is removed to show the internal components.

The CIBER-2 instrument, ${ }^{9-11}$ depicted in Figure 2, consists of a $\mathrm{LN}_{2}$-cooled Cassegrain telescope with a 28.5 $\mathrm{cm}$ aperture, combined with lens optics, dichroic beamsplitters, and three $2048 \times 2048 \mathrm{HgCdTe}$ Hawaii-2RG* focal plane detector arrays. The selected 4" pixel size yields an instantaneous FOV of $2.3^{\circ} \times 2.3^{\circ}$. The assembly housing the lens optics is constructed from aluminum alloy 6061-T6, and the lenses are made from anti-reflection coated S-FPL51, S-FPL53, S-LAL8, S-TIH6, S-BSL7, and silica glass. The assembly is carefully designed to maintain optical alignment and focus through launch acceleration and vibration. Dichroic beamsplitters and Windowpane filter are used to obtain three wide-field images in six photometric $1.1^{\circ} \times 2.3^{\circ} \mathrm{FOV}$ bands. Additionally, a small portion of each focal plane includes a linear variable filter (LVF) with continuous $\mathrm{R}(=\lambda / \Delta \lambda)=20$ spectral coverage over 0.4 square degrees to measure the absolute EBL from $0.5-2 \mu \mathrm{m}$. A schematic of the focal plane assembly is shown in Figure 3. The aluminum housing is hard black anodized to reduce reflections inside the telescope assembly. The popup baffle is stowed upon deployment and re-entry, and extend during observations to shield the optics from thermal radiation from the skin and the Earth. The instrument is designed to record dark frames prior to launch, and again on ascent and descent, using a cold shutter placed just behind the primary mirror at an optical pupil. We have designed a calibration source mounted behind the secondary mirror to provide a known illumination in each band using a set of LEDs, giving a transfer standard before and during flight.

The CIBER-2 instrument was integrated into a Black Brant IX sounding rocket at Wallops Flight Facility in Virginia, and will be flown multiple times from White Sands Missile Range in the New Mexico desert. The first

\footnotetext{
* Manufactured by Teledyne Scientific \& Imaging, LLC.
} 
flight is projected for early 2021.

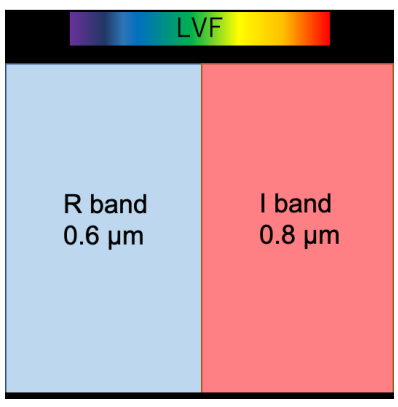

(A)

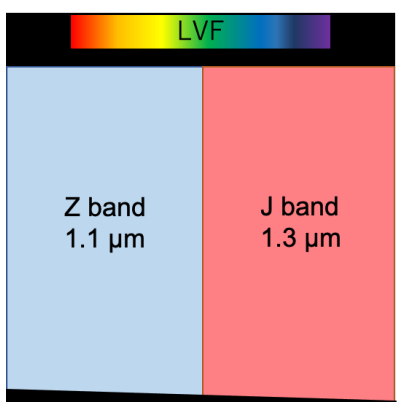

(B)

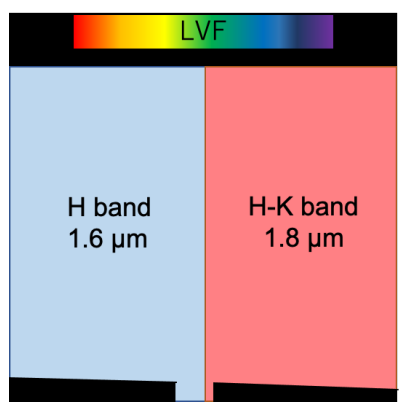

(C)

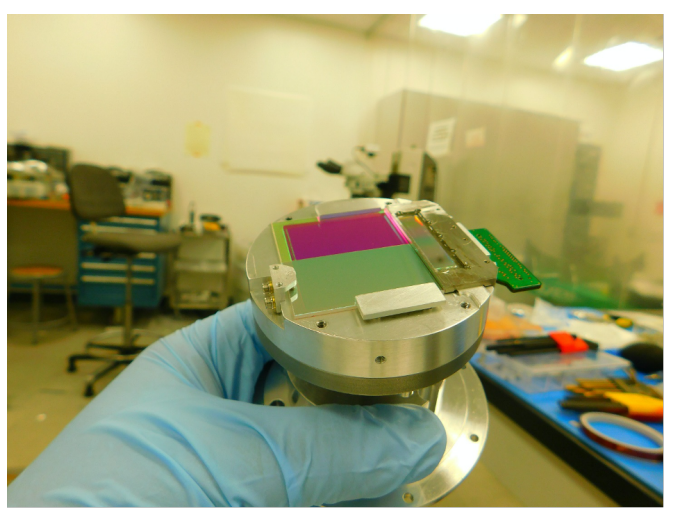

(D)

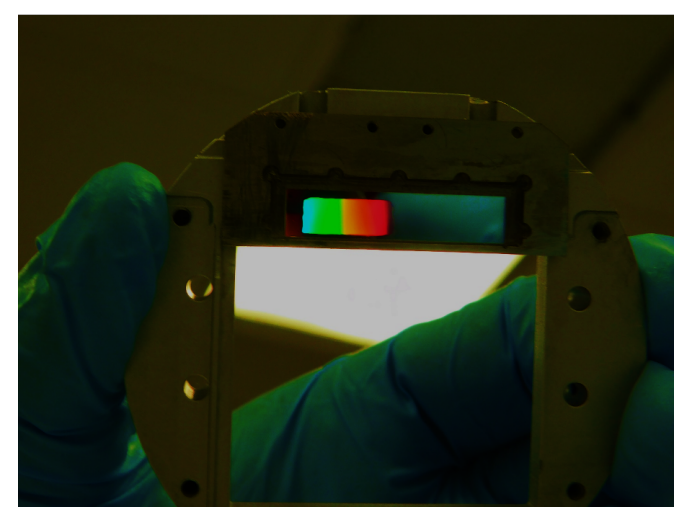

(E)

Figure 3. A: Schematic of the focal plane filter assembly for Arm-S. B: Schematic of the focal plane filter assembly for Arm-M. C: Schematic of the focal plane filter assembly for Arm-L. D: Photograph of the focal plane filter assembly. E: Photograph of the LVF.

\section{PRE-FLIGHT PERFORMANCE EVALUATION}

CIBER-2 is currently qualified for 1st flight. We have completed a variety of pre-flight optical tests, which we use to make focus adjustments, spectral response measurements, and absolute photometric calibrations. In this section, we discuss the methods behind these tests and their results for pre-flight performance evaluation.

\subsection{Focus Adjustments}

The detector positions are adjusted with shims to account for movement of the focal planes due to heat contraction. A schematic of the setup for focus scan with collimator is shown in Figure 4. The pinhole position on the collimator is moved by stepper motor assembly. For each detector, we performed broad focus scans at three
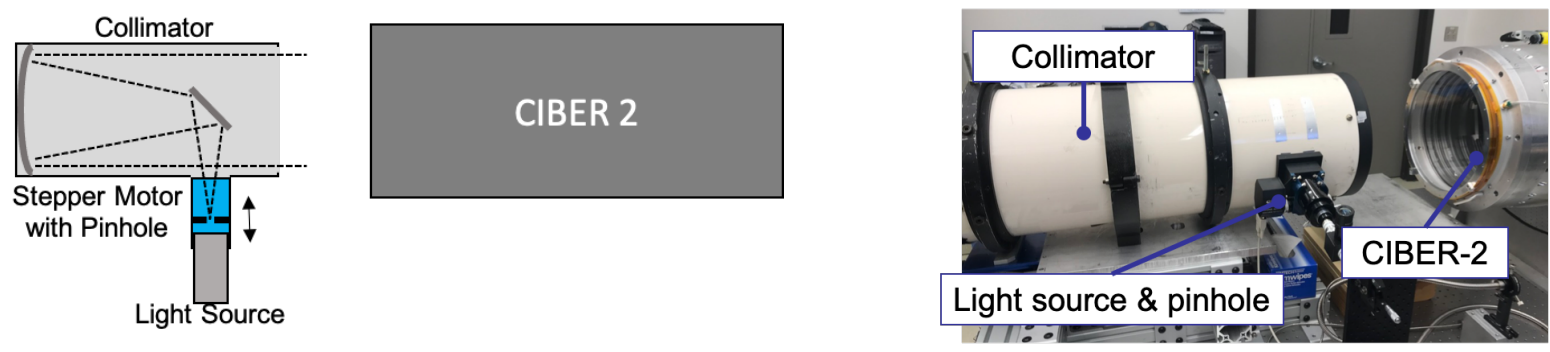

Figure 4. Schematic and photograph of setup for the focus scan with collimator. 
detector positions and tilt scans near the best focus at three other positions. Image data was taken by Ground Station Electronics system. ${ }^{12}$ The scan locations are provided by optics simulation. The mean of absolute shifts from the collimator focus and the scan locations on the focal plane is shown in left side of Figure 5. The error is the $95 \%$ confidence interval for the Gaussian curve fit, which may underestimate the actual uncertainty. Donutlike structure at the center of the Arm-M is due to thermal radiation of room temperature through the leak of the longwave cut filter. In Arm-L, in-band thermal emission was shown in the right Windowpane and longwave region of LVF. The measured point-spread function (PSF) size by collimator focus scan is also shown in right
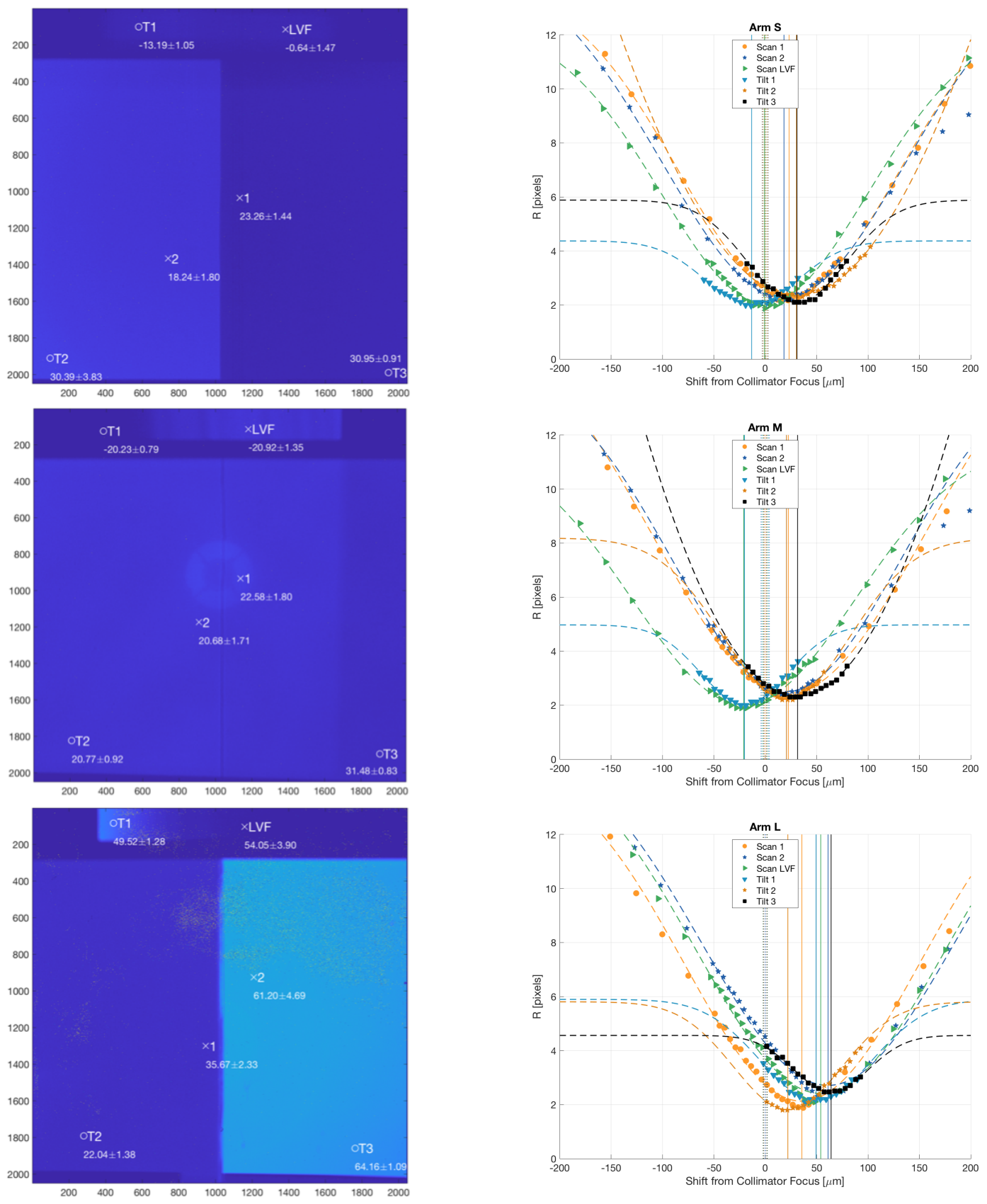

(A)

(B)

Figure 5. A: The mean of absolute shifts from the collimator focus and the scan locations on the focal plane. B: PSF size $R$ by collimator focus scan. Mean of absolute shift for Arm-S is $19.44 \mu \mathrm{m}$ (top), for Arm-M is $22.78 \mu \mathrm{m}$ (middle), and for Arm-L is $47.77 \mu \mathrm{m}$ (bottom). 
side of Figure 5. The radius of the measured PSF at the best focus is 2 pixels for all detectors, which is small enough for CIBER-2 observation.

\subsection{Integration Testing}

CIBER-2 was integrated at Wallops Flight Facility in summer 2019 and underwent additional vibration testing in early 2020 with NASA-provided payload equipment for a sequence of flights. This integration tested the operation of the arrays and electronics with the telemetry and power systems. The entire payload went through the usual battery of environmental and sequence testing to reach flight readiness. After the integration campaign the instrument was shipped to White Sands Missile Range in the New Mexico desert.

We verified the focus position and best focus PSF size before and after pre-flight vibration testing. Photograph of the state at the time of payload vibration test is shown in Figure 6(A). From the inspection of the PSF images, no significant difference of the best focus PSF sizes from pre- and post-vibration is seen. For the focus position, there was a slight shift caused not by the CIBER-2 optics but presumably by the collimator measurement system because the PSF size looks same. Figure 6(B) shows the result of payload vibration test in summer 2019. ${ }^{\dagger}$ We will verify the tested optics at White Sands Missile Range in 2021 during a launch campaign.

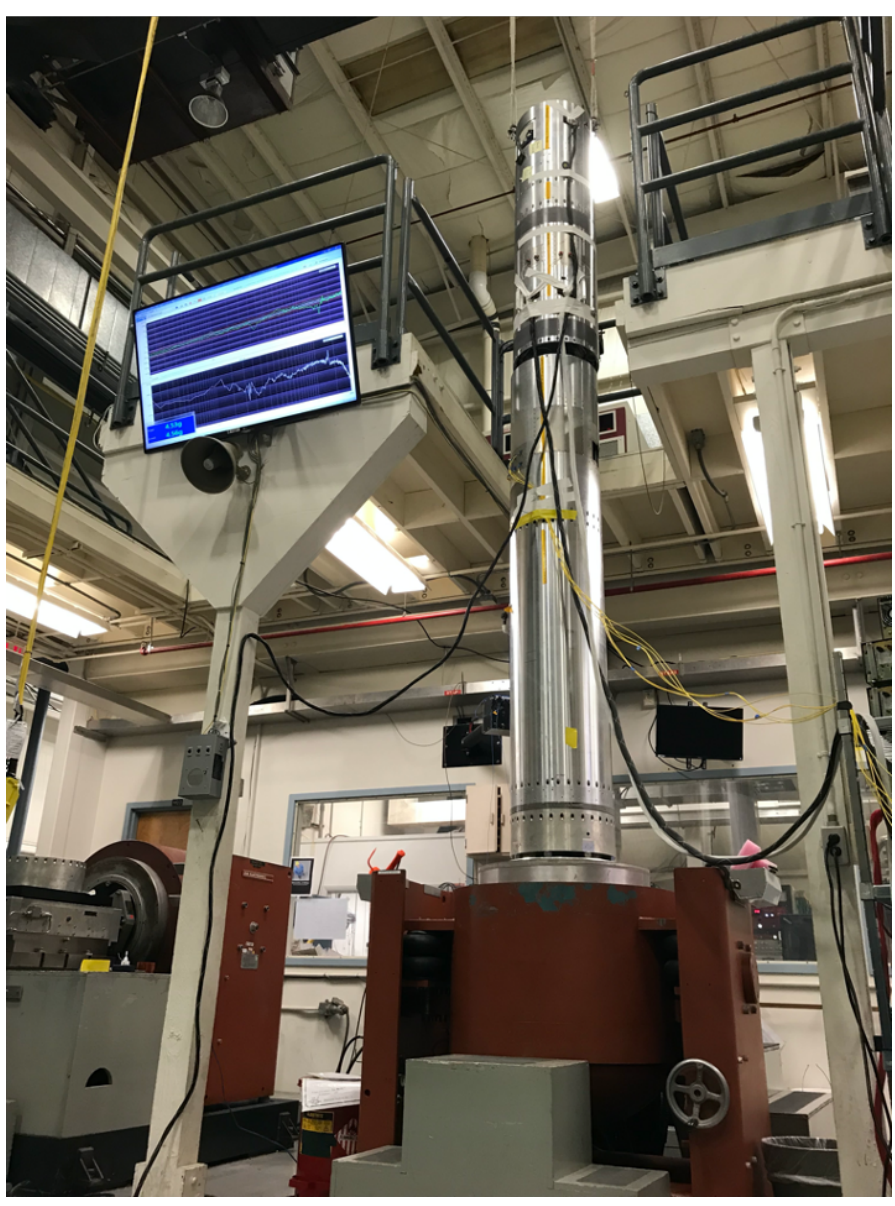

(A)
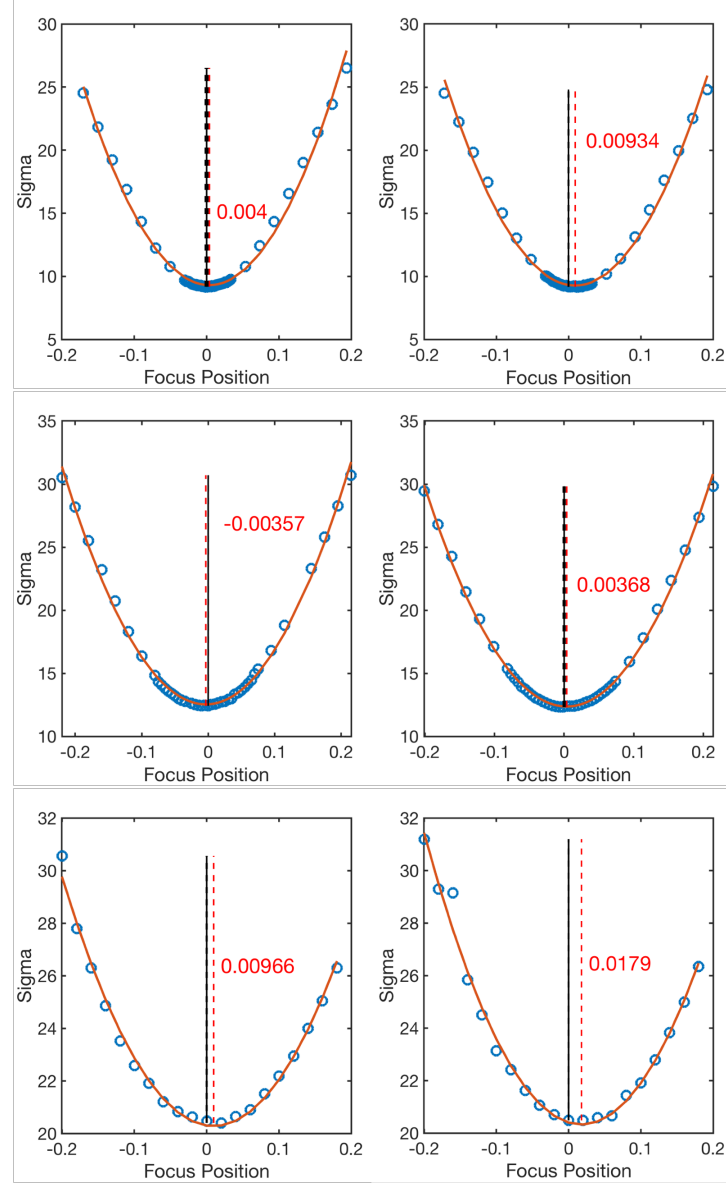

(B)

Figure 6. A: Photograph of the payload during the 2019 vibration test. B: Focus position and best focus PSF size between before (left) and after the vibration test. None of the focus positions changed significantly from the vibration test.

${ }^{\dagger}$ Figure 6(B) uses a different profile than Figure 5 due to the different testing equipment, but is well suited to show that there is no change in PSF size before and after vibration. 


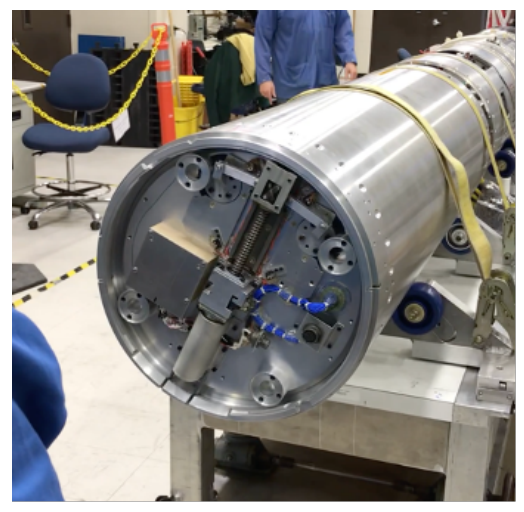

(A)

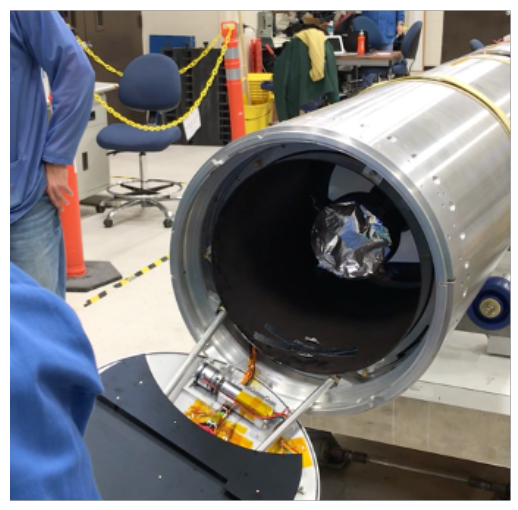

(B)

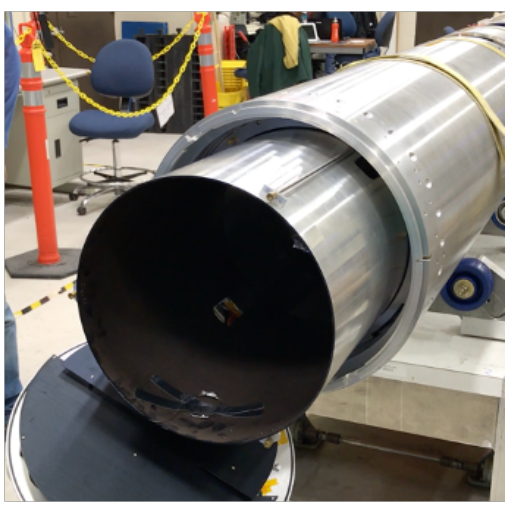

(C)

Figure 7. Photograph of flight sequence testing of shutter door and popup baffle operations. A: Shutter door, in closed position. Mechanisms for opening and closing the shutter door visible on the outside of the door in closed position. B: Shutter door, in open position. There are In-flight control wiring and motor mechanism on lower half of door panel for popup baffle control. Popup baffle is still stowed. C: Popup baffle was fully deployed.

We also tested the sequence of shutter door and popup baffle operations before and after the vibration test. The popup baffle extends upon deployment to block stray light from the rocket skin, open shutter door, and the Earth (see Section 3.5). Figure 7 shows the flight sequence test of shutter door and popup baffle with the telemetry and power systems. To verify the baffle performance at cold temperature, we used a cryogenic vacuum chamber mated to the payload's shutter door to test the door and baffle's operation. We found that the door and the baffle's performance at $<100 \mathrm{~K}$ is consistent with room temperature.

\subsection{Spectral Response Measurements}

In order to measure the spectral response of individual pixels in each focal plane, we measured the pixelto-wavelength calibration using a scannable monochromatic light source. A schematic of the setup for the wavelength calibration and spectral resolution measurement is shown in Figure 8. CIBER-2 views the aperture of the integrating sphere while the wavelength of the monochromator is varied from 450 to $1000 \mathrm{~nm}$ in $10 \mathrm{~nm}$ increments for Arm-S. Similarly, it is varied from 940 to $1500 \mathrm{~nm}$ in $10 \mathrm{~nm}$ increments for Arm-M, and 1400$2100 \mathrm{~nm}$ in 10-20 nm increments for Arm-L. The wavelength resolution of the monochromator is $15 \mathrm{~nm}$ in this measurement, which is smaller than the LVF resolution. Figure 9 shows the relation between photo current and
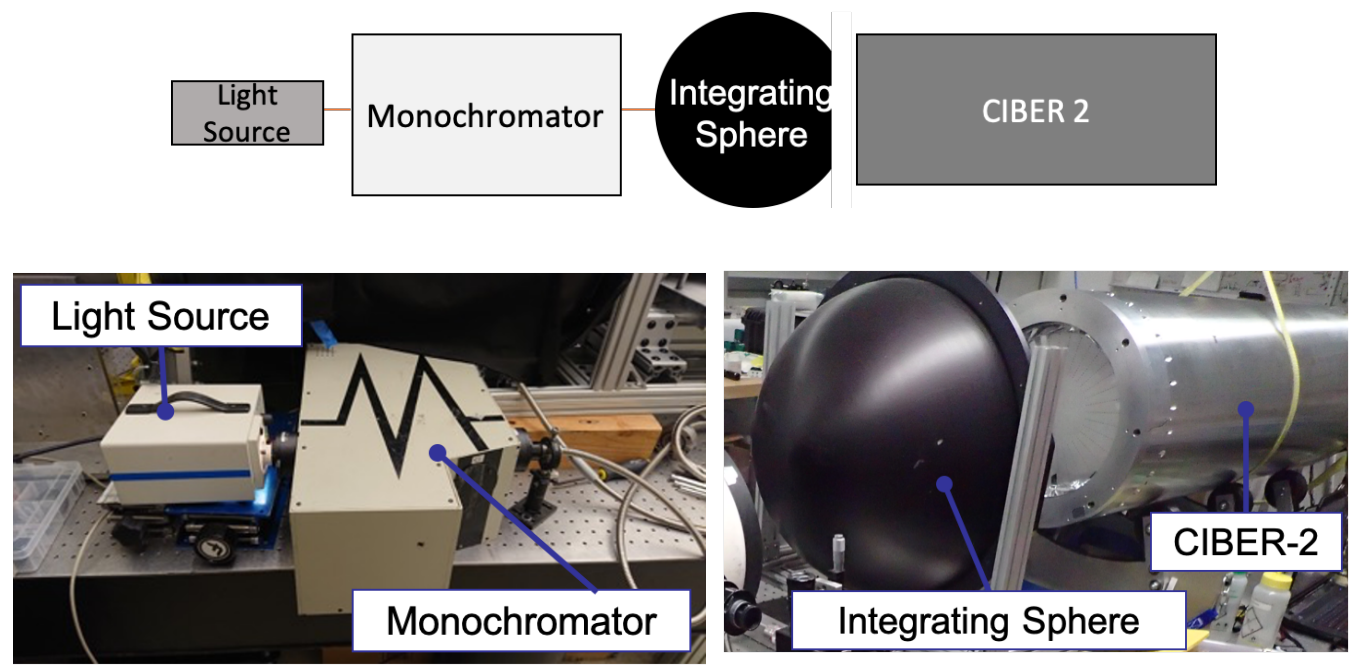

Figure 8. Schematic and photograph of setup for the pixel-to-wavelength calibration and spectral resolution measurement. 
wavelength of LVF (left) and Windowpane filter (right). For LVF, based on this result, the relation between wavelength and pixel was derived, and pixel-to-wavelength relation was linear as expected. In addition, Figure 10 shows the measured wavelength resolution $\mathrm{R}(=\lambda / \Delta \lambda)$ of LVFs from spectral resolution measurement, which is found to be consistent with the design specifications $\mathrm{R} \sim 20$. The measured spectral resolution is nearly identical across the three LVFs.
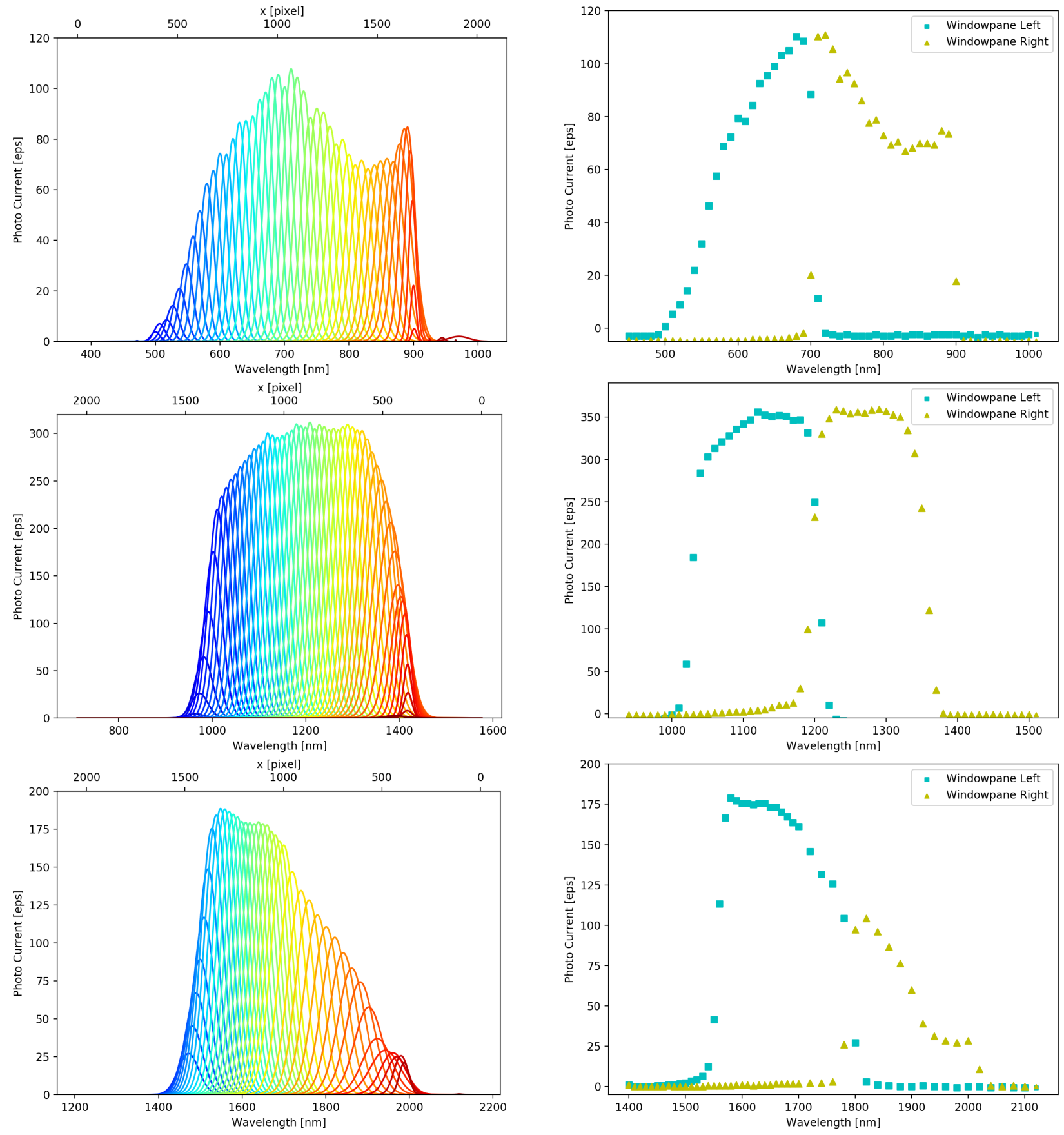

Figure 9. The pixel-to-wavelength calibration for Arm-S (top), Arm-M (middle), and Arm-L (bottom). Relation between photo current and wavelength of LVF (left) and Windowpane filter (right). 


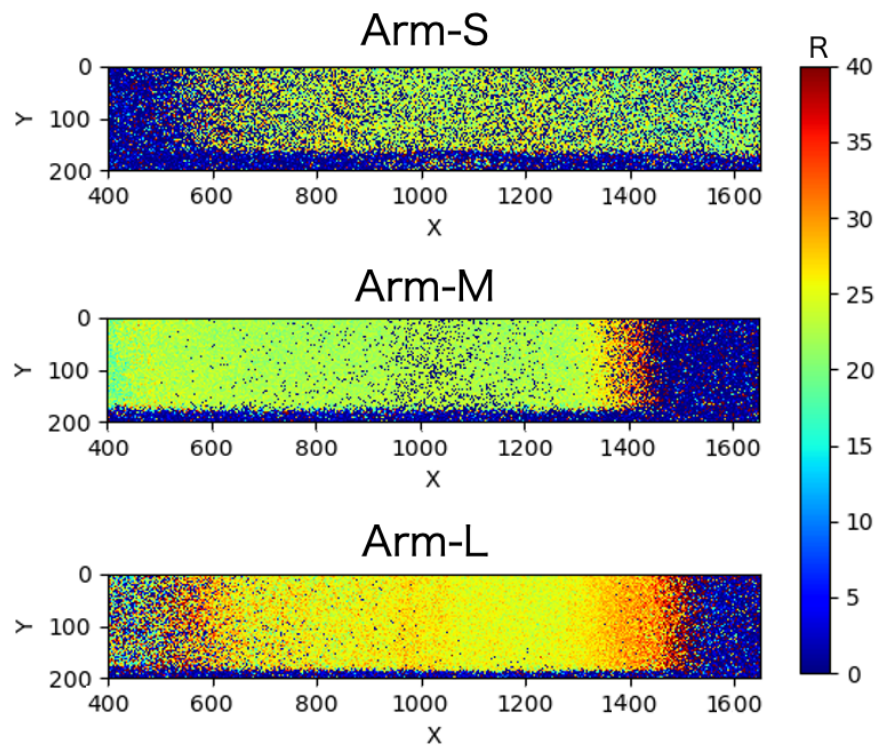

Figure 10. LVF Wavelength resolution maps. All arms satisfied the resolution requirement of $\mathrm{R} \sim 20$.

\subsection{Absolute Photometric Calibrations}

Since the primary scientific motivation for the CIBER-2 is to determine the absolute spectrum of the EBL, absolute photometric calibration is an essential component of the CIBER-2 instrument characterization. In particular, CIBER-2 requires an absolute calibration to extended emission. Absolute photometric calibration is performed by using diffuse light sources of two kinds. The optical calibration is done with a Tungsten-Halogen lamp with an effective temperature of $2800 \mathrm{~K}$, fiber-coupled to a series of integrating spheres through a fiber in-line attenuator module. Absolute radiance at the sphere output is measured with an absolutely calibrated spectrometer. A schematic of the setup using a Tungsten-Halogen source is shown in Figure 11. Since the sphere aperture is smaller than the CIBER-2 aperture, we employ an aperture stitching method which uses a sectorial
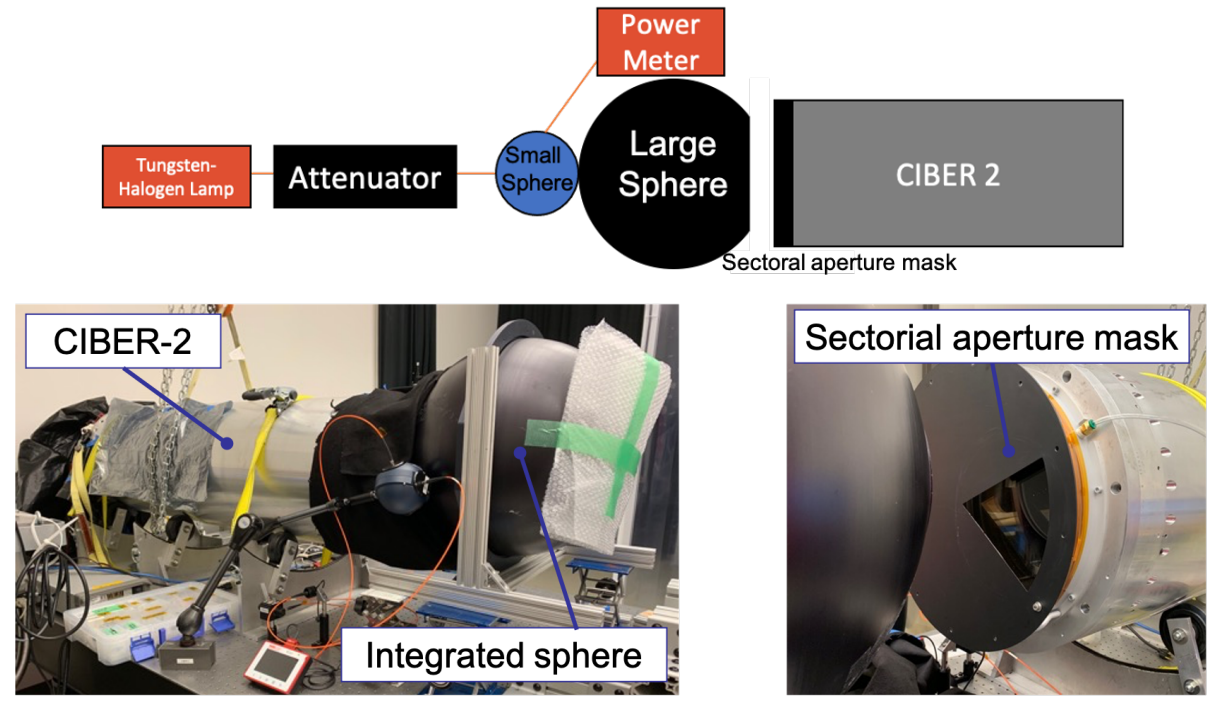

Figure 11. Schematic and photograph of setup for the visible band calibration. 
aperture mask (shown in Figure 11). The relationship between absolute radiance $I(\lambda)\left[\mathrm{nW} / \mathrm{m}^{2} / \mathrm{sr} / \mathrm{nm}\right]$ and the detector's signal, also known as photo current $S\left[\mathrm{e}^{-} / \mathrm{sec}\right]$, can be described as

$$
S=I(\lambda) \cdot C
$$

where $C$ is the conversion factor $\left[\left(\mathrm{e}^{-} / \mathrm{sec}\right) /\left(\mathrm{nW} / \mathrm{m}^{2} / \mathrm{sr} / \mathrm{nm}\right)\right]$, and is given by:

$$
C=\frac{\lambda \eta}{h c} A_{t e l} \Omega_{p i x} T_{o p t} \Delta \lambda
$$

where $\eta$ is a quantum efficiency, $h$ is Planck's constant, $c$ is the speed of light, $A_{t e l}$ is the aperture of CIBER-2, $\Omega_{p i x}$ is a field of view of a pixel, $T_{o p t}$ is transmittance of optics, and $\Delta \lambda$ is a spectral band width. Figure 12(A) shows the preliminary measurement of conversion factor for Arm-S. Based on this measurement, the detection limit of Arm-S at the $5 \sigma$ confidence level for a $0.2\left[\mathrm{e}^{-} / \mathrm{sec}\right]$ readout noise and a $50 \mathrm{~s}$ integration is estimated as shown in Figure 12(B). For LVF, it was calculated for single pixel and 5000 pixels, and for Windowpane filter, it was calculated for single pixel and 850000 pixels, respectively.

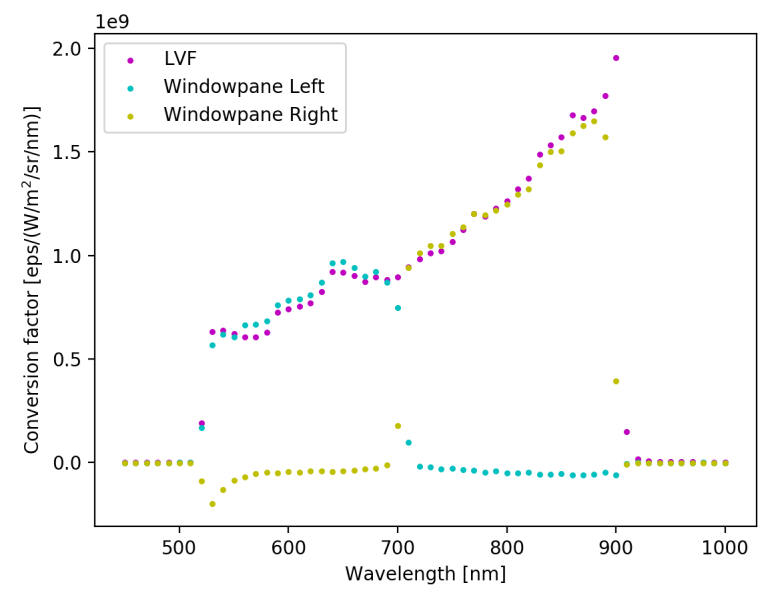

(A)

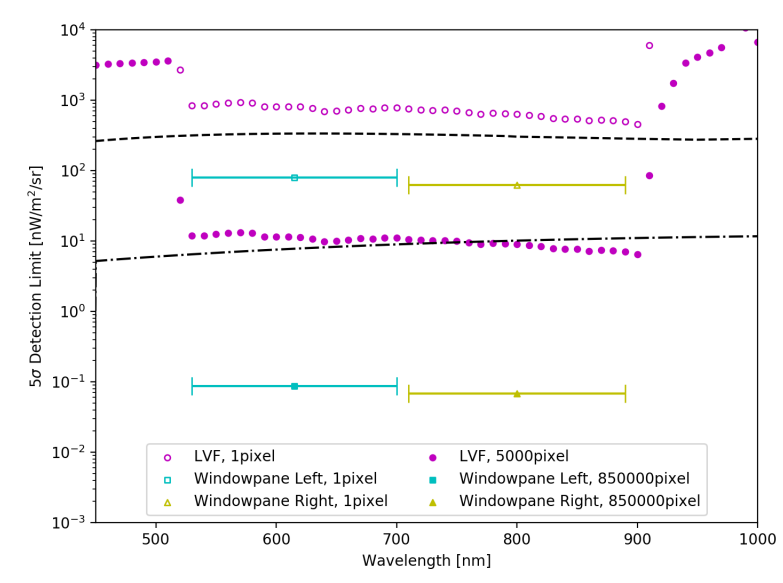

(B)

Figure 12. A: Preliminary measurement of conversion factor for Arm-S. B: $5 \sigma$ detection limit of Arm-S for 50 s estimated from the readout noise. The dotted line shows the Zodiacal Light measured by CIBER, ${ }^{13}$ and the dash-dot line shows the Integrated Galactic Light model. ${ }^{14}$
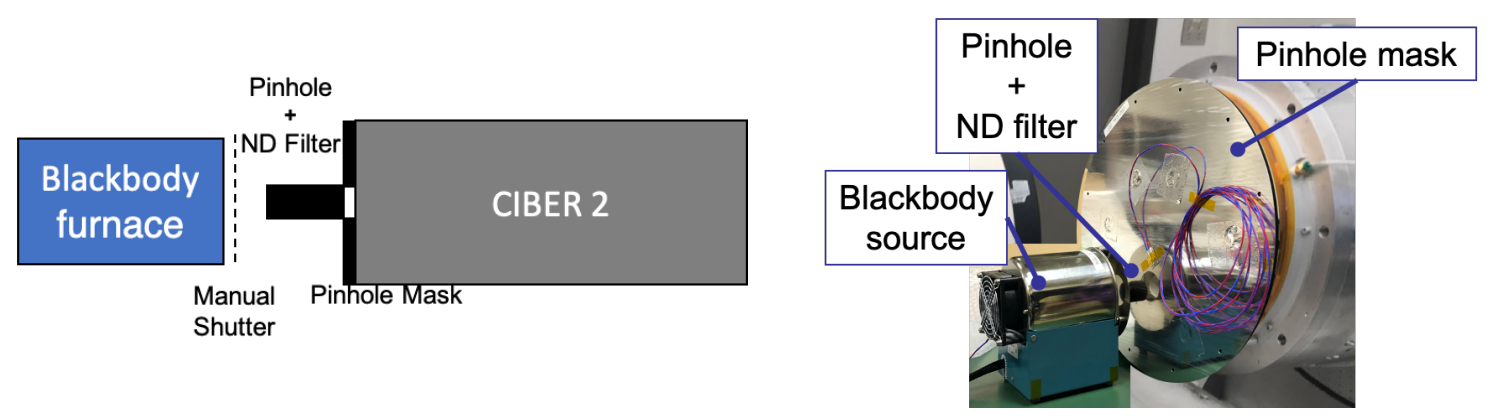

Figure 13. Schematic and photograph of setup for the near-infrared bands calibration.

Another source is a standard blackbody source at $1200 \mathrm{~K}$ with a pinhole aperture and Neutral Density (ND) filter stack for relative flux calibration in the near-infrared bands, and the absolute radiance is scaled by using the wavelength overlap with the visible band calibration. A schematic of the setup using a blackbody source is 
shown in Figure 13. Since the aperture of blackbody source with a pinhole is smaller than the CIBER-2 aperture, an aperture stitching method with a pinhole mask is used. Maximum acceptable charge is $\sim 10^{4}$ electrons for a requirement to achieve $1 \%$ accuracy of the absolute calibration. We have already selected the best ND filter for calibration and it will be ready to be conducted in the United States.

\subsection{Baffle Performance Estimates}

The popup baffle extends beyond the end of the rocket skin after opening the shutter door, and is designed to block stray emission paths from the rocket skin, the shutter door, and the Earth. We estimate the stray light from the Earth with the baffle response function derived based on the signal response from numerical calculation of responsivity for point sources outside the CIBER-2 FOV. ${ }^{6,15}$ Figure 14(A) shows the estimated value of the baffle performance simulation in terms of the baffle response function, which is defined as

$$
g(\theta)=\frac{4 \pi}{\Omega} G(\theta)
$$

where $\Omega$ is the solid angle of the detector and $G(\theta)$ is the normalized response to a point source, quantifying sensitivity to off-axis sources in surface brightness measurements. It is independent of the field of view. $g(\theta)$ is derived from two rough fittings. Intensity of the integrated stray light contribution from Earth's radiation in surface brightness unit is given by:

$$
I_{\text {stray }}\left(\theta_{\text {Earth }}\right)=\frac{1}{4 \pi} \int g(\theta) I_{\text {Earth }}(\theta, \phi) d \Omega
$$

where $\theta_{\text {Earth }}$ is the angle between the Earth rim and the CIBER-2 pointing direction, and $I_{\text {Earth }}(\theta, \phi)$ is the surface brightness of the Earth, assumed as the Equation (5).

$$
I_{\text {Earth }}(\theta, \phi)=\left\{\begin{array}{l}
6 \times 10^{4}\left[\mathrm{nW} / \mathrm{m}^{2} / \mathrm{sr}\right] \\
0(\text { out of the Earth) }
\end{array}\right.
$$

The Equation (4) was calculated numerically and the result is shown in Figure 14(B) as a function of the angle from the Earth. Since the minimum elevation angle in CIBER-2 observation targets is $>60 \mathrm{deg}$, the estimated stray intensity from the Earth is negligible.

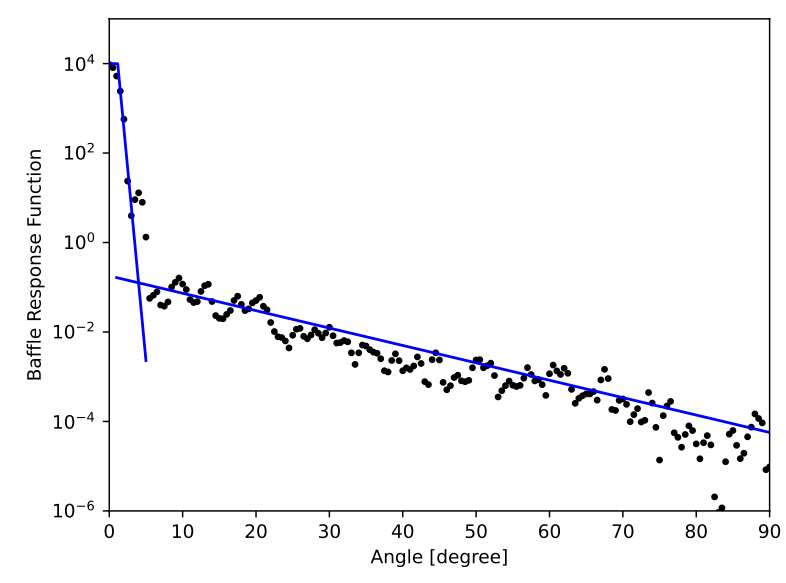

(A)

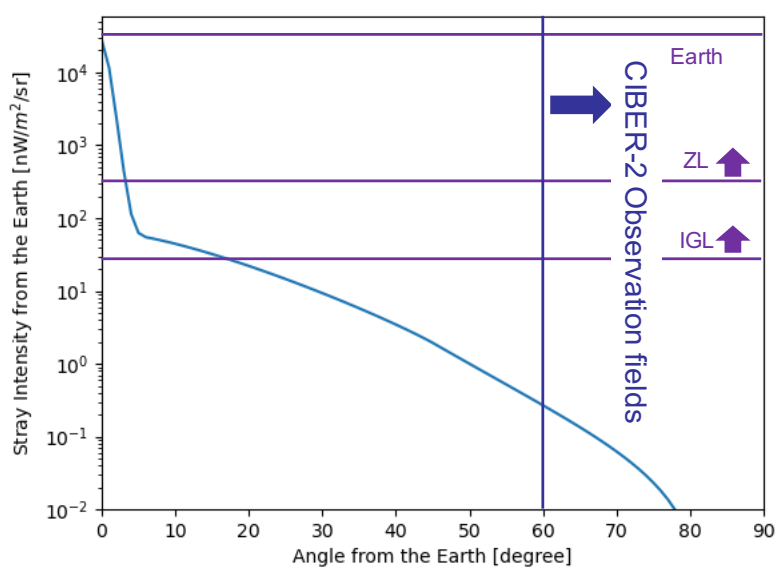

(B)

Figure 14. A: The baffle response function by an off-axis response simulation. B: The estimated stray intensity from the Earth to the CIBER-2. Brightness of the Zodiacal Light and the Integrated Galactic Light are also shown. 


\section{CONCLUSIONS}

The primary scientific motivation for the CIBER-2 is to determine the absolute spectrum of the EBL and to define the source of the EBL excess fluctuation. CIBER-2 is currently qualified for 1st flight from White Sands Missile Range, scheduled for early 2021. We evaluated the optical performance of the CIBER-2 instrument in the laboratory as described in this paper and found the following:

1. The detector position was adjusted with an accuracy of $\pm 50 \mu \mathrm{m}$ to minimize the measured PSF sizes as a whole over the detector area.

2. Using data from before and after vibration tests, we measured the best-fit PSF to be sufficiently small for CIBER-2 observations.

3. Pixel-to-wavelength relation was consistent with the linear fit, and the wavelength resolution of LVFs was consistent with the design prediction $\mathrm{R} \sim 20$.

4. The conversion factor for the visible band was derived by absolute photometric calibration. The nearinfrared band calibration is ready to conduct at WSMR.

5. The stray light signal from the Earth is negligible for the range of angles observed in flight using the popup baffle.

\section{ACKNOWLEDGMENTS}

CIBER-2 was supported by NASA APRA research grants NNX07AI54G, NNG05WC18G, NNX07AG43G, NNX07AJ24G, and NNX10AE12G. Japanese participation was supported by KAKENHI (2034, 18204018, 19540250, 21340047, 21111004, and 15H05744) from Japan Society for the Promotion of Science (JSPS) and the Ministry of Education, Culture, Sports, Science and Technology (MEXT). Korean participation was supported by the Pioneer Project from Korea Astronomy and Space Science Institute (KASI). C.H.N was supported by NASA Headquarters under the NASA Earth and Space Science Fellowship Program - Grant 80NSSCK0706. We would like to thank the NASA Wallops Flight Facility engineers and staff for their technical supports.

\section{REFERENCES}

[1] Cooray, A., Bock, J. J., Keatin, B., Lange, A. E., and Matsumoto, T., "First star signature in infrared background anisotropies," The Astrophysical Journal 606(2), 611 (2004).

[2] Zemcov, M., Smidt, J., Arai, T., Bock, J., Cooray, A., Gong, Y., Kim, M. G., Korngut, P., Lam, A., Lee, D. H., et al., "On the origin of near-infrared extragalactic background light anisotropy," Science 346(6210), $732-735$ (2014).

[3] Cooray, A., Smidt, J., De Bernardis, F., Gong, Y., Stern, D., Ashby, M. L., Eisenhardt, P. R., Frazer, C. C., Gonzalez, A. H., Kochanek, C. S., et al., "Near-infrared background anisotropies from diffuse intrahalo light of galaxies," Nature 490(7421), 514-516 (2012).

[4] Kashlinsky, A., Arendt, R., Ashby, M., Fazio, G., Mather, J., and Moseley, S., "New measurements of the cosmic infrared background fluctuations in deep spitzer/irac survey data and their cosmological implications," The Astrophysical Journal 753(1), 63 (2012).

[5] Matsumoto, T., Seo, H., Jeong, W.-S., Lee, H., Matsuura, S., Matsuhara, H., Oyabu, S., Pyo, J., and Wada, T., "Akari observation of the fluctuation of the near-infrared background," The Astrophysical Journal $\mathbf{7 4 2}(2), 124$ (2011).

[6] Tsumura, K., Matsumoto, T., Matsuura, S., Sakon, I., and Wada, T., "Low-resolution spectrum of the extragalactic background light with the akari infrared camera," Publications of the Astronomical Society of Japan 65(6), 121-121 (2013).

[7] Madau, P. and Pozzetti, L., "Deep galaxy counts, extragalactic background light and the stellar baryon budget," Monthly Notices of the Royal Astronomical Society 312(2), L9-L15 (2000). 
[8] Totani, T., Yoshii, Y., Iwamuro, F., Maihara, T., and Motohara, K., "Diffuse extragalactic background light versus deep galaxy counts in the subaru deep field: missing light in the universe?," The Astrophysical Journal Letters 550(2), L137 (2001).

[9] Lanz, A., Arai, T., Battle, J., Bock, J., Cooray, A., Hristov, V., Korngut, P., Lee, D. H., Mason, P., Matsumoto, T., et al., "Studying extragalactic background fluctuations with the cosmic infrared background experiment 2 (ciber-2)," in [Space Telescopes and Instrumentation 2014: Optical, Infrared, and Millimeter Wave], 9143, 91433N, International Society for Optics and Photonics (2014).

[10] Shirahata, M., Arai, T., Battle, J., Bock, J., Cooray, A., Enokuchi, A., Hristov, V., Kanai, Y., Kim, M. G., Korngut, P., et al., "The cosmic infrared background experiment-2 (ciber-2) for studying the near-infrared extragalactic background light," in [Space Telescopes and Instrumentation 2016: Optical, Infrared, and Millimeter Wave], 9904, 99044J, International Society for Optics and Photonics (2016).

[11] Nguyen, C. H., Stewart, B., Bang, S.-C., Bock, J. J., Cooray, A., Danbayashi, K., DeSantiago, A., Hristov, V., Kojima, T., Korngut, P., et al., "Integration and instrument characterization of the cosmic infrared background experiment 2 (ciber-2)," in [Space Telescopes and Instrumentation 2018: Optical, Infrared, and Millimeter Wave], 10698, 106984J, International Society for Optics and Photonics (2018).

[12] Park, W.-K., Bang, S.-C., Battle, J., Bock, J., Cooray, A., Danbayashi, K., DeSantiago, A., Hristov, V., Kojima, T., Korngut, P., et al., "Development of data storage system and gse for cosmic infrared background experiment 2 (ciber-2)," in [Space Telescopes and Instrumentation 2018: Optical, Infrared, and Millimeter Wave], 10698, 1069849, International Society for Optics and Photonics (2018).

[13] Matsuura, S., Arai, T., Bock, J. J., Cooray, A., Korngut, P. M., Kim, M. G., Lee, H. M., Lee, D. H., Levenson, L. R., Matsumoto, T., et al., "New spectral evidence of an unaccounted component of the nearinfrared extragalactic background light from the ciber," The Astrophysical Journal 839(1), 7 (2017).

[14] Finke, J. D., Razzaque, S., and Dermer, C. D., "Modeling the extragalactic background light from stars and dust," The Astrophysical Journal 712(1), 238 (2010).

[15] Bock, J., Sullivan, I., Arai, T., Battle, J., Cooray, A., Hristov, V., Keating, B., Kim, M., Lam, A., Lee, D., et al., "The cosmic infrared background experiment (ciber): the wide-field imagers," The Astrophysical Journal Supplement Series 207(2), 32 (2013). 